\title{
Pleomorphic Adenoma of Small Salivary Glands
}

\section{Open Access Journal of Dental and Oral Surgery (OAJDOS)}

\section{Volume 2 Issue 2, 2021}

Article Information

Received date : August 06, 2021

Published date: August 16, 2021

\section{*Corresponding author} Vladimíra Schwartzová, Clinic of Stomatology and Maxillofacial Surgery of the Faculty of Medicine at the University Pavol Jozef Šafárik and Louis Pasteur University Hospital, Slovakia

\section{Keywords}

Benign salivary gland tumors; pleomorphic adenoma; pleomorphic adenoma of small salivary glands

\section{Distributed under Creative Commons} CC-BY 4.0

\author{
Vladimíra Schwartzová ${ }^{1}$, Jozef Jendruch ${ }^{1}$, Milan Béreš ${ }^{1}$ Róbert Luby ${ }^{1}$ and Peter \\ Kizek $^{2}$ \\ ${ }^{1}$ Clinic of Stomatology and Maxillofacial Surgery of the Faculty of Medicine at the University Pavol Jozef \\ Šafárik and Louis Pasteur University Hospital, Slovakia \\ ${ }^{2}$ Head of Clinic of Stomatology and Maxillofacial Surgery of the Faculty of Medicine at the University Pavol \\ Jozef Šafárik and Louis Pasteur University Hospital, Slovakia
}

Summary

In their work, the authors focus on the pleomorphic adenoma of small salivary glands, its characteristics, location, and occurrence. They describe some cases of its rare occurrence and unusual location. In the conclusion, they emphasize the prevention of possible malignancy of this type of benign tumor.

\section{Introduction}

Most benign salivary gland tumors are found in the parotid salivary gland. The average age of patients with benign salivary gland tumors is 42 years. With malignant tumors the average age of a patient is 53 years. The incidence of malignancies is higher in small salivary glands than in big salivary glands [1]. Whereas adenoma growing from small salivary glands in the oral cavity is rare [2]. Adenomas are benign epithelial tumors of the salivary glands. The WHO classification lists up to 9 types of adenomas. Pleomorphic adenoma, also called mixed tumor or myxochondroepithelioma, is the most clinically significant adenoma. It grows slowly, sometimes to considerable proportions. It usually manifests itself only in asymptomatic swelling. It has an incomplete capsule and protrusions emerge from it into the surrounding tissue. If the principles of proper surgical treatment are not maintained, the tumor relapses and the risk of malignant transformation increases [3]. Up to $90 \%$ of mixed tumors total incidence is in the parotid gland. In terms of histological composition, mode of development and shape, they are similar to small salivary gland tumors. However, due to their location, the clinical signs as well as the method of surgical treatment differ [2]. On imaging methods, they appear as well-defined round masses, which on ultrasound appear as hypoechogenic formations and on MRI as homogeneous brightening [4].

Material and Methodology

In our work, we present two cases of pleomorphic adenoma of small salivary glands. The first case is a 35 -year-old patient with a formation on the left side of the hard palate. The patient observes this formation for about 6 months (Figure 1). The solid consistency was without fluctuation, did not bleed or hurt. The formation has grown in the last 2 months. The formation was extirpated and the defect was covered with a rotating lobe. The material was sent for histological examination (Figures 2-5). The second case is a 74-year-old patient who has had a rigid formation in the oral cavity for about 20 years. The size of the formation limits her quality of life (Figure 6). All teeth were extracted in the jaw, she never wore a total replacement. The formation is placed on the right side of the mucosa of the mxilla's alveolar ridge. It is large. Changes in terms of oppressive resorption are visible on OPG X-ray (Figure 7). In both cases, radical surgical treatment with histologization was performed.

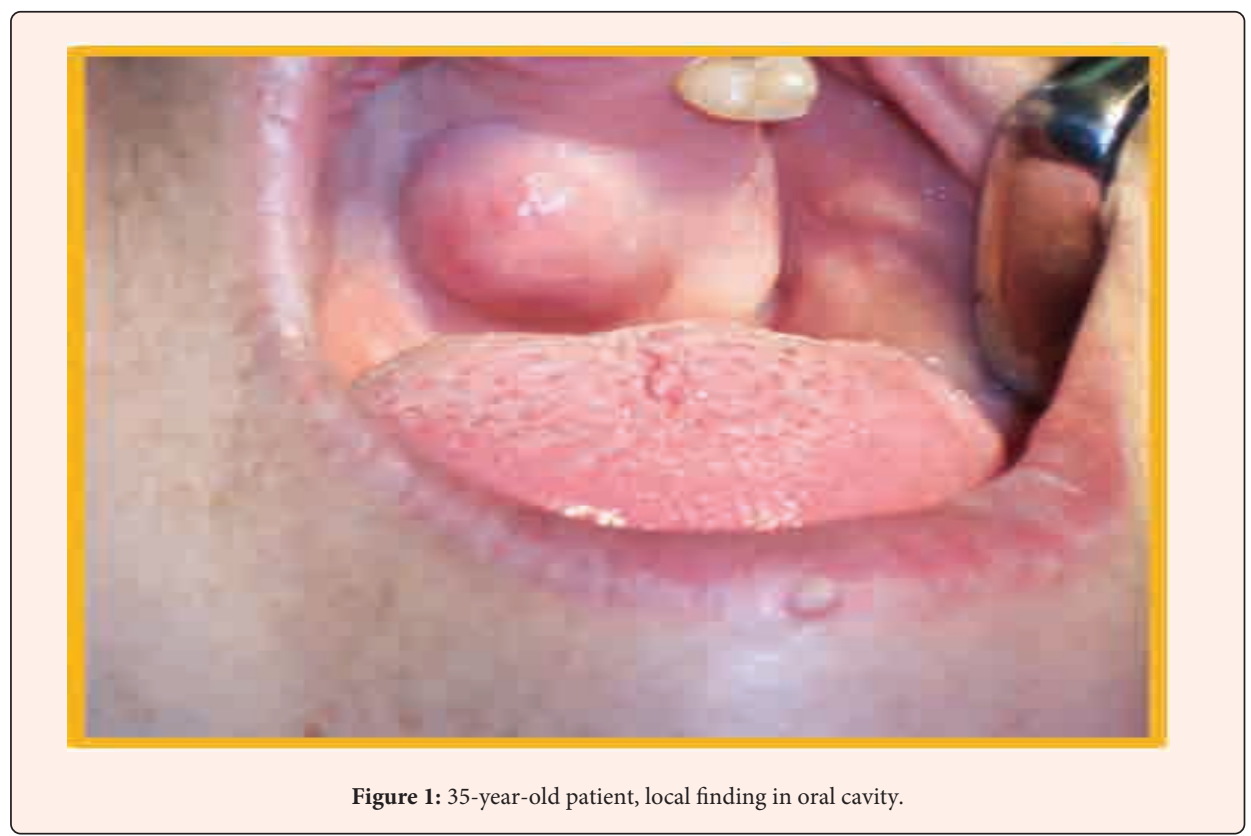




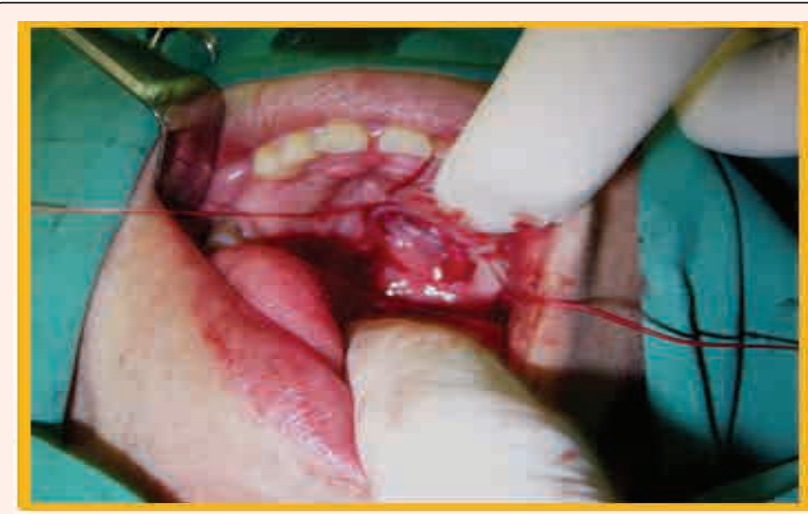

Figure 2: Perioperative image, excision of the formation, coverage of the defect with a rotating mucosa lobe.

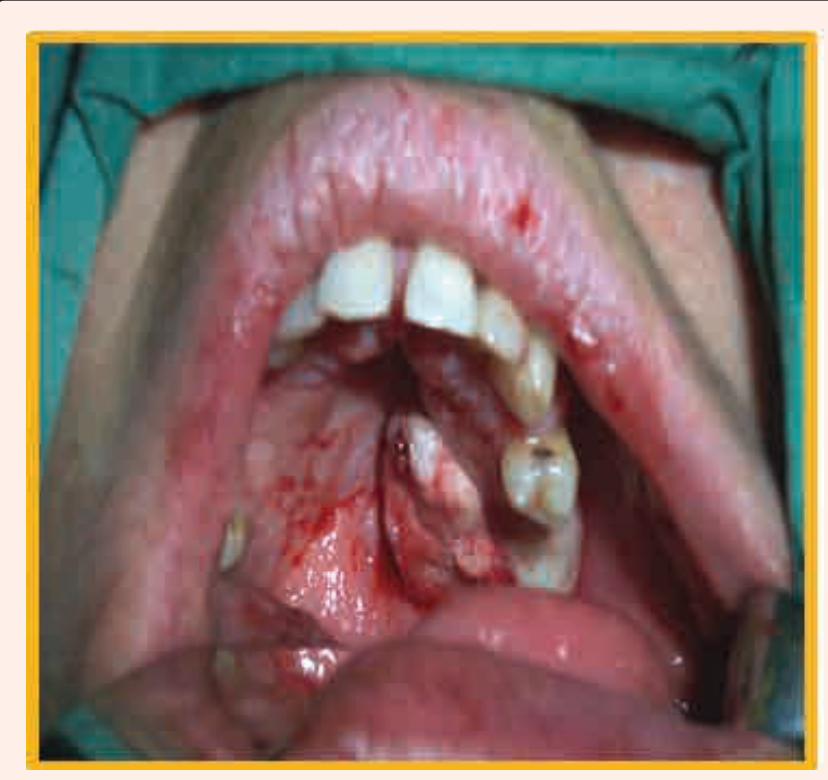

Figure 3: Perioperative image, knotting over a tampon.

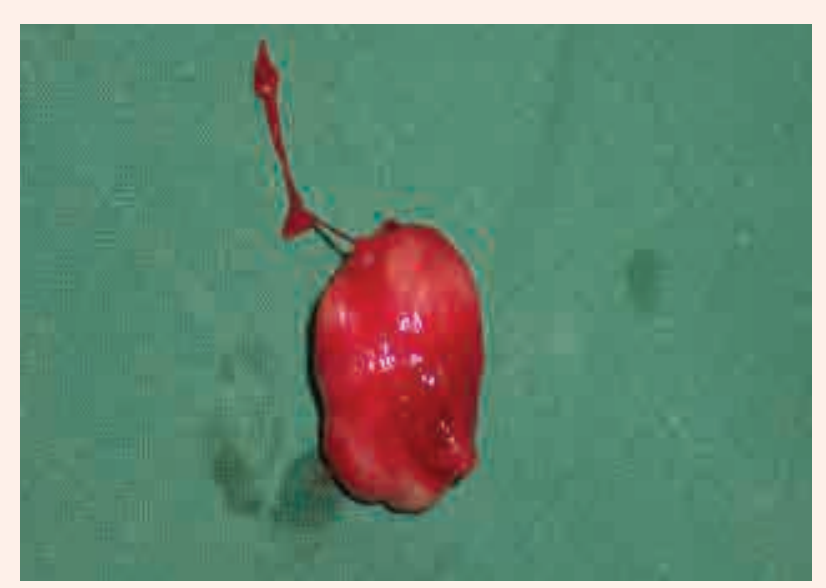

Figure 4: Extirpated formation.

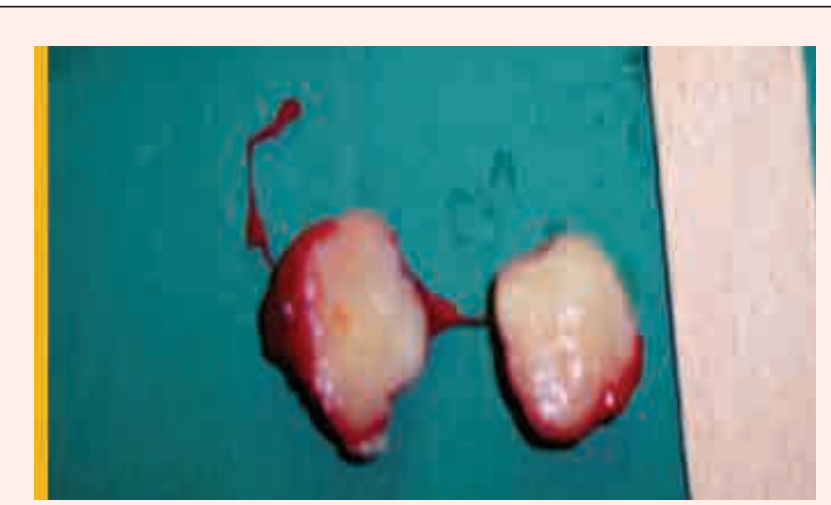

Figure 5: Cross cestion of the extirpated formation.

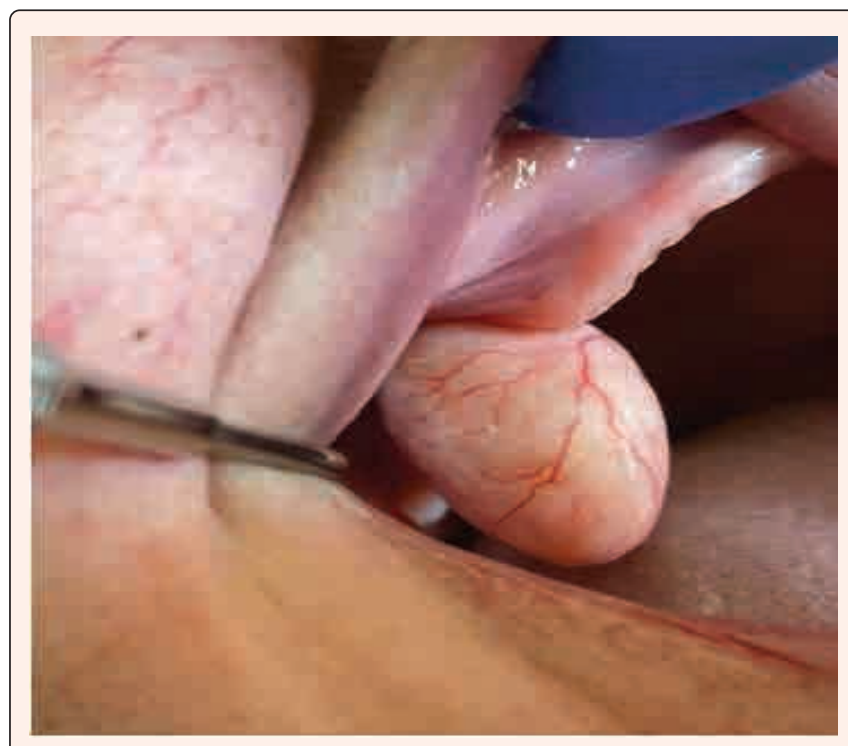

Figure 6: 74-year-old patient, local finding in the oral cavity.

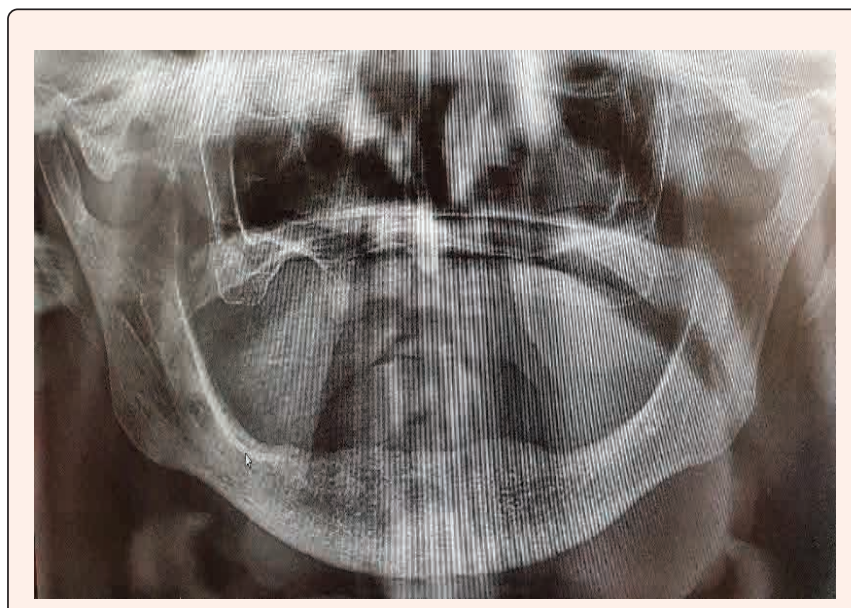

Figure 7: 74-year-old patient, OPG X-Ray image. 


\section{Discussion}

The pleomorphic adenoma growing from the small salivary glands is most often located in the palate or on the buccal mucosa. It occurs in younger people between 20 and 30 years of age, but it can also occur in the elderly. This is shown also by the two cases selected by us above. The pleomorphic adenoma is well demarcated, encapsulated and stored submucosally. It is usually round, has a smooth surface and is elastic. Cyst, torus palatinus, fibroma, chondroma, adamantinoma and sarcoma come into consideration in differential diagnosis. The exact diagnosis can be determined only on the basis of histological examination. In the microscopic image, the tumor consists of epithelial foci and bands embedded in a sparse stroma of myxomatous to cartilaginous appearance. The treatment is surgery. If the clinical diagnosis is uncertain, perioperative rapid incision provides the necessary information and influences the choice of an adequate surgical solution. Along with the tumor, part of the adjacent bone and mucosal structures are resected with sufficient radicality [1]. The prognosis is good, but the patient should be monitored long-term.

\section{Conclusion}

Even benign tumors can grow infiltratively and metastasize. On the other hand, some malignant tumors have a good prognosis. The WHO classification lists up to 17 types of salivary gland carcinomas. The incidence of malignancies is increasing towards the small salivary glands [1]. The prognosis of patients after surgical treatment of pleomorphic adenoma is good, but the patient should be monitored long-term. Even in this case, faster treatment means a better prognosis.

\section{References}

1. Longauerová I (1995) Tumors of the salivary glands. In Černý J, et al. (Ed.), Special Surgery, Neck and Head Surgery. Martin: Osveta, pp. 452-454.

2. Toman J (1976) Oral and maxillofacial surgery. $2^{\text {nd }}$ (edn.) Prague: Avicenum, Czech Republic, pp. 229-230.

3. Wotke J (2001) Pathology of the orofacial area. Praha: Grada Publishing, Czechia, pp. 336.

4. Saber M, Gailard F. Pleomorphic adenoma of salivary glands. 